Creative Potential is Differentially Expressed in School, at Home, and the Natural Environment

\author{
Mark A. Runco \\ Southern Oregon University
}

Ahmed M. Abdulla Alabbasi

Arabian Gulf University

Selcuk Acar
University of North Texas

Alaa Eldin A. Ayoub

Aswan University

Arabian Gulf University

Correspondence concerning this article should be addressed to Mark A. Runco, $P h D$, Director of Creativity Research \& Programming, Southern Oregon University, 1250 Siskiyou Blvd, Ashland, OR 97520. Email: RuncoM@sou.edu

Declaration of Interest: We wish to confirm that there are no known conflicts of interest associated with this publication and there has been no significant financial support for this work that could have influenced its outcome.

Funding: This research did not receive any specific grant from funding agencies in the public, commercial, or not-for-profit sectors. 


\begin{abstract}
Creative potential is one of the very most important topics for research. It is difficult to study because, unlike creative products, potential is by definition latent. There are several methods. One involves comparing creative activity expressed in various settings. Previous research has, for example, compared creativity expressed in school with that expressed by the same individuals when they are in the natural environment (i.e., outside of school). There tends to be more creative activity outside of school, suggesting that individuals have creative potential but it is only allowed to be expressed in certain settings. The present investigation extended this line of research by comparing creative activity in school, at home, and that occurring outside of school and home. The comparison of three settings implies that the current investigation is relevant to the "press" category of creativity research. Results of the present investigation indicated that the activity scores from the three settings shared less than $52 \%$ of their variance. The measures used were highly reliable, so the conclusion was that, as in previous research, various settings do indeed differentially allow the expression of creative potential. Comparisons of means also supported this finding. Interestingly, creative activity at home was significantly more common than creative activity at school and when outside of the home and school. A statistical test of method variance indicated that it was not a notable contribution nor confound. Limitations are discussed at the end of the article.
\end{abstract}

Keywords: Creativity gap; Creative Activity and Accomplishment, Settings of Creativity, Ideational Behavior, Attitudes and Values 


\section{Creative Potential is Differentially Expressed in School, at Home, and the Natural Environment}

The field of creative studies has grown tremendously in the past few decades, yet the exact meaning of "creativity" is still debated (Acar et al., 2017; Simonton, 2012; Weisberg, 2015, 2021). One suggestion is to avoid the noun creativity and use the adjective instead. This leads to more precise wording (e.g., creative process, creative achievement, creative product, creative economy). It also fits well with the 4P framework (Rhodes, 1961) which distinguishes among process, product, place, and person. Simonton (1990) added persuasion to the 4Ps, the idea being that creative people and creative things change the way that others think. Runco (2007) offered a hierarchical framework with creative potential and creative performance at the top level and all the other "Ps" subsumed under one of those two broad categories.

In addition to offering a clear distinction about avoiding the ambiguity of the noun "creativity," the top two categories of the hierarchical framework help when studying one of the most important questions in the field: (a) how to identify creative potential and (b) how to support it (so it develops into actual creative performance. There are quite a few different methods designed to fulfill creative potentials (reviewed by Runco, in press). Each assumes that humans have creative potential. After all, if there is no potential (an unlikely prospect), efforts to enhance creativity are bound to fail. If there is potential, there is room for growth, even if the individual is not yet actually performing in a manifestly creative fashion. Some efforts to support creativity are programmatic and systematic (Ma, 2006, 2009). The fulfillment of creative potential also depends on informal education, family background (Abdulla Alabbasi et al., 2021), culture, and various other things that are not programmatic.

Given the importance of the two questions about (a) identification and (b) fulfillment, it 
is surprising that there is so little research on creative potential per se. One line of research has examined creative potential by comparing how it is expressed in different settings. The idea here is that individuals have creative potential and certain settings allow its expression, while other settings do not. In a sense this line of work contributes to both our understanding of potential, but also "press" and the impact of setting on that potential. In some of the earliest research on this topic, Holland (1961) reported differences between school and extracurricular creativity. He developed the Creative Activity and Accomplishment Checklist (CAAC) and administered it, along with various academic measures, to several thousand high school juniors. The CAAC is a self-report that asks how often the student has been involved in various creative pursuits and how often awards have been won in various creative domains. True, it is a self-report, so things like memory and honesty may influence scores, but then again, as Hocevar and Bachelor (1989) pointed out, the individual is the best informed about his or her has own activities and accomplishments. Further, past creative activity and accomplishment are predictive of future creative activity and accomplishment (Hocevar, 1981). The CAAC also has the advantage of being domain specific: it may cover 8 or even 10 different domains (e.g., science, math, art, music writing, technology, leadership or social recognition, moral creativity). Various validation studies support the use of the CAAC (e.g., An \& Runco, 2015; Paek \& Runco, 2017; Runco, 1986; Runco et al., 1990). It is not dissimilar to measures more recently described by Jauk et al. (2012) and Kaufman (2012). Holland and Richards (1965) reported that extracurricular activities and achievements were at best moderately correlated with academic performances.

Runco et al. (2016) extended this line of work by comparing CAAC performances in school with creative performance displayed outside of school. Their methodology improved on that used by Holland and Richards (1965), and of course it was worthwhile to reexamine the 
relationship of extracurricular activity and academic achievement, given that over 50 years have passed. Runco et al. reported that more creative activities and accomplishments occurred outside of school compared with those occurring when in school. A second study, also using the CAAC, confirmed that more creative activity occurs when outside of school compared with that occurring when in school (Runco et al., 2017). Runco et al. (2017) were concerned with this "creativity gap" because they felt that it implied that there was wasted creative potentialpotential expressed when individuals are not in school but not expressed when they are in school.

The present investigation extended this line of work in several ways. The primary objective was to compare three settings, namely school, home, and the natural environment (i.e., "outside," meaning neither home nor school). The relationship of school and home creative activity with outside creative activity was of special interest, given that school and home had been studied previously. Other objectives included examining relationships of the CAAC domain scores with measures of Creative Attitudes and Values, Creative ideation, and a fairly new selfreport asking respondents "where are you the most creative?" Results from this investigation will contribute to our understanding of how different settings have an impact on creative potential and where and why creative potentials are the most or least likely to be expressed.

\section{Method}

\section{Participants}

Six hundred and forty-one middle and high school students were recruited from the Kingdom of Bahrain $(n=225)$ and the United Arab Emirates $(n=415)$, of which 50.1\% was female. The mean age of the participants was $14.98 \mathrm{yrs}(S D=1.85)$. Most students attended private schools (92.4\%)-a factor taken into account in analyses. Parents of 211 participants (32.9\%) participants held a Master's or Doctoral degree; 194 (30.3\%) held a high school degree; 
133 (20.7\%) held an undergraduate degree; 88 (13.7\%) finished middle school; and 15 (2.3\%) finished only elementary school. Participants' family size ranged between 3 to 9 members, with $40.4 \%$ being from families with 5-6 members. Ninety-nine participants (15.4\%) reported that their family consisted of more than 9 members.

Instruments

Creativity Activities and Accomplishment Check List (CAAC). Four domains of the CAAC were used in this study: Art (nine items), Math and Science (seven items), Technology (nine items), and Everyday Creativity (seven items). Previous studies showed that the CAAC had good reliability, with Cronbach's alphas ranging from .74 to .88 (Runco et al., 2016, 2017). The directions for the CAAC were as follows:

"The next question asks how often you experienced certain things. For each question, check the answer(s) that best describes what you have done in the past 2-3 years. It may be necessary to approximate. The response options allow you to tell us if you have done each thing (1) at home, (2) at school, or (3) outside of home and school (i.e., you have done it but neither at home nor at school). You may choose more than one answer. You may have done some things both at home and at school, for example. Here are the options:

(A) Never at school = you have never done the activity or accomplishment at school; (B) Once or twice at school = you have done this activity or accomplishment once or twice at school;

(C) Three or more $=$ you have done this activity or accomplishment 3-5 times at school;

(D) Never at home = you have never done the activity or accomplishment at home;

(E) Once or twice at home = you have done this activity or accomplishment once or twice 
at home;

(F) Three or more = you have done this activity or accomplishment 3-5 times at home;

(G) Never outside of school or home = you have never done the activity or accomplishment outside the school or home;

(H) Once or twice outside of school or home $=$ you have done this activity or accomplishment once or twice outside the school or home;

(I) Three or more $=$ you have done this activity or accomplishment 3-5 times outside the school or home.

Each item began with "How of often have you...." and then the particular activity was named.

Attitudes and Values (A\&V). A children's version of the A\&V, with 24 items, was used in the current study because it was quite possible that attitudes about creativity influence the individual's involvement in creative activity. Previous studies that employed an A\&V measure showed high reliability (Acar \& Runco, 2014). Participants were first shown an example of how to respond to each of the $\mathrm{A} \& \mathrm{~V}$ items, after which the actual 24-items were presented. A "smiley" response scale was used, instead of a traditional Likert-type scale. The response options are given below. The total score was calculated by averaging all 24 items of the $\mathrm{A} \& \mathrm{~V}$. The directions for the A\&V were as follows:

"Use the smiley scale (below) to tell us how much you agree or disagree with questions 1-24. This is fun: you slide the bar from one smiley to the next until it shows how much you agree or disagree. The smiley face will change as you move the bar. It goes from a frown, which means that you do not agree at all, all the way up to a big smile, which means that you completely agree. Remember, for each question, slide the bar to choose 
the face that shows how much you agree. Here are the options:

Totally disagree $=$ This is something I completely disagree with

Mostly disagree $=$ This is something I do not agree with

Neutral $=$ I do not agree or disagree with this

Mostly agree $=$ This is something I agree with

Totally agree $=$ This is something I completely agree with

Where are you Creative (WAYC)? This instrument was developed by Runco et al.

(2017) to assess students' perceptions of where they believe they are creative. Those perceptions might be related to the actual creative behavior that is assessed by the CAAC. The WAYC consists of 15 creativity-related adjectives (self-expressive, concern about being weird or odd, innovative, inventive, flexible, independent/autonomous, aware of expectations and sociallyappropriate behavior, playful, original/novel/unique, creative, unconventional, spontaneous, authentic-true to yourself and who you really are, thinking of new ideas, and exploratory). Each adjective is accompanied by a five-point Likert type scale (below) which allows the respondent to indicate where they display each behavior. The directions for the WAYC were as follows:

"The list of labels in the following questions (1-15) may describe you, at least some of the time, or they may not describe you at all. Use the scale (below) to tell us where you are most likely to show each behavior. Here are the options for your responses:
(A) Mostly at home--I am this way when I am at home.
(B) Mostly at school--I am this way when I am at school.
(C) Both at home and school--I am this way when I am at home and at school.
(D) Some other place--I am only this way when I am not at home or school
(E) I am never this way 
Choose only one option from (A) to (E)."

WAYC subscale scores were based on a count of (1) how many WAYC items were assigned by the respondent to the Home (when asked "where are you the most likely to be..."); (2) how many WAYC items were assigned to School; and (c) How many WAYC items were assigned to Outside.

Runco Ideational Behavior Scale (RIBS). The RIBS was developed by Runco et al. $(2000,2001)$ to measure ideation. It is an estimate of general (i.e., not domain specific) creative potential. As discussed above, the expression of potential (as it varies from setting to setting) was one of the key concerns of this research. A response scale ranging from "0 - never" to "4 very often" was used. In the current study, a short form consisting of 25 items was administered. The RIBS score used in the analyses was an average across these 25 items. Information about discriminant validity, construct validity, and reliability for the original instrument, with US samples, are reported in Plucker, Runco, and Lim (2006) and Runco et al. (2000, 2001). Considering the Arab sample, a previous study showed that the reliability coefficient was .90 when a 50-item RIBS was administered to college level students (Runco et al., 2016). The directions for the RIBS are as follows:

"Use the 'smiley' scale (below) to tell us how often each of the sentences describes you or your thinking and behavior. Please tell us how you really think and behave, not how you think you are supposed to behave. This is fun! You choose the smiley face that is closest to how you feel by sliding the bar next to it. The smiley face will change as you move the bar. You can slide the bar to tell us that you never behaved that way, or you behave that way very often, or something in between.

\section{Procedure}


The study protocol was prepared by the second author and submitted to the Scientific Research Directorate of the Ministry of Education in the Kingdom of Bahrain and the United Arab Emirates. Once approval was granted all instruments were uploaded to the SoGoSurvey website (https://survey.sogosurvey.com/r/SvLWsf). Study participants were identified by school administrations, and all middle and high school students who were interested in participating in this study were contacted via email. The three main schools that participated in the current study were: (a) Dubai Modern Education School (UAE), (b) the Ibn Khulood National School (Bahrain), and (c) Abdul Rahman Kanoo International School (Bahrain).

The first page of the online survey included the consent letter, followed by demographic information (school name, parents' education, age, gender, school type, and number of people living in the home). After that they measures were administered in the following order: A\&V, WAYC, CAAC, and then the RIBS.

\section{Results}

The reliability of the scales was calculated. All three CAAC scores were reliable: Home $($ alpha $=.91)$, School $($ alpha $=.92$, and Outside $($ alpha $=.94)$. The primary objective of this research was tested with a multiple regression. Given what was said above about relationships with the Outside scale being of special interest the first regression used the CAAC Outside scale as criterion and the School and Home CAAC scales as predictors. Parental education, type of school (private or public), sex, and age were entered in a first step of the regression (each dichotomized, except age) as control variables. Results indicated that the control variables accounted for a small $(2 \%)$ but statistically significant amount of variance $\left(R^{2}=.021, p=.040\right)$. The second step of this analysis, entering the CAAC School and Home scales, was also statistically significant $\left(R^{2}=.557, p<.001\right)$. Examination of the equation (weights) indicated 
that none of the individual control variables was statistically significant in the first step of the regression, but age, sex, and parental education were significant in the second step, as part of the final solution $(\beta \mathrm{s}=.548,3.02,-3.17$, respectively, $t \mathrm{~s}(606)=2.53,3.93$, and $-2.74, p \mathrm{~s}=.012, .001$, .006). The weights for the CAAC School and CAAC Home indices in the final equation were both statistically significant $(\beta \mathrm{s}=.517$ and .323 , respectively, $t \mathrm{~s}(606)=7.53$ and 12.51 , both $p \mathrm{~s}$ $<.001)$.

Given the possibility of method variance, we examined the variance inflation factor values, which were within acceptable range (<2.30) (Hair et al., 1995; Kock, 2015; Rogerson, 2001). More is said about method variance in the Discussion. Bivariate correlations among the three CAAC scales were .721 (Home-School), .635 (Home-Outside), and .700 (School-Outside), all $p \mathrm{~s}<.001$. Thus, the scales shared less than $50 \%$ of their variance.

Another way to look at the various CAAC scores (Home, School, Outside) involves the examination of means. It is one thing to look at shared variance using multiple regression, but additional information can be obtained with paired $t$-tests using to compare the averages. These $t$-tests indicated that the mean of the CAAC Home score $(M=28.49, S D=12.64)$ differed significantly from the mean of the Outside CAAC score $(M=17.43, S D=13.78 ; t(640)=24.70$, $d=.98, p<.001)$; the mean of the Home CAAC score differed significantly from the School CAAC score $(M=24, S D=13.08 ; t(640)=11.81, d=.47, p<.001)$; and the mean of the School CAAC score differed significantly from the Outside CAAC score $(t(640)=15.96, d=.63, p<$ $.001)$. The effect sizes for those three comparisons were $.97, .47$, and .63 , respectively. They indicate that creativity activity (and accomplishment) was the highest at Home followed by School and Outside.

\section{Canonical Correlation}


A canonical correlational analysis was performed to examine relationships between the three CAAC scores and (a) Creative Attitudes and Values, (b) RIBS, and (c) three subscales of the Where Are You Creative measures. The criteria in the canonical analysis were the Home, School, and Outside CAAC total scores. This analysis indicated that there were strong correlations between the predictors and the three CAAC scores. Indeed, three orthogonal variates were uncovered $(R \mathrm{c}=.484, .261$, and .094, Wilks $=.766(F(15,1748)=15.56, \mathrm{p}<.001$, for the first variate, $.924(F(8,1268)=6.41, \mathrm{p}<.001$, for the second variate, $.991(F(3,635)=1.90, p=$ .128 , for the third variate. The first variate explained $23.4 \%$ of the variance, the second variate added $6.8 \%$ to the variance explained, and the third variate, which was not significant, added only $0.9 \%$, taking the total of explained variance by the three predictors to $31.1 \%$.

Other information about these relationships is provided by the bivariate correlations presented in Table 1. These also indicated that the shared variance of the three CAAC scales was significant but below $52 \%(.635<r \mathrm{~s}<.721)$. The bivariate correlations also indicate that, of the three predictors, the RIBS was the most strongly related to the three CAAC scores $(.351<r \mathrm{~s}<$ .419 , all $p \mathrm{~s}<.01)$. The Creative Attitudes and Values measure was also positively and significantly related to the three CAAC scores $(.159<r \mathrm{~s}<.201$, all $p \mathrm{~s}<.01)$, though not as highly as the RIBS scores.

\section{Insert Table 1 about here}

\section{Discussion}

This investigation offers important information about how creative activities and accomplishments vary from setting to setting (i.e., Home, School, and the Natural environment, or what we called "outside"). Results indicated that there was overlap among the three settings, but differences as well. Overlap was expected, given that all three CAAC indices focused on 
creativity, but the overlap was far from $100 \%$. Indeed, shared variance was mostly below $50 \%$. Differences were confirmed with paired $t$-tests. These indicated that the individuals in this sample reported significantly more creative activities at Home than when in the other two settings.

The correlational analyses confirmed that there was some overlap among settings, which was entirely expected, given that all three measures focused on the creativity of the students. Yet only $52 \%$ of the variance in the Home and School scores was shared. This of course left $48 \%$ of the variance. Some of that could reflect measurement error, though the reliabilities of the three CAAC scores were quite good, so measurement error seems to have been minimal. "Method variance" could have contributed to overlap among the three CAAC scores but the variance inflation factors values $(<2.3)$ were lower than the pathological levels of 3.3. Thus, this was probably not an issue in our data (cf. Kock, 2015). In addition, method variance would have increased the shared variance, thus making it higher than it was in actuality. Thus, if method variance was a factor, the true shared variance would have been lower than the $52 \%$ found and the relationships among Home, School, and Outside would be even lower than the coefficients reported here. Thus, we can say that the creative performances of the student in the three areas overlapped, but the overlap was moderate at best. This is entirely consistent with the previous investigations summarized above (Runco et al., 2016, 2017). So again, creativity seemed to vary from setting to setting.

Runco et al. (2017) interpreted the differences they found (the "creativity gap") as indicating that students have creative potential and that this potential is expressed more easily in some settings (outside of school) than other settings (the school). This interpretation implies that schools may be overlooking or even wasting the creative potentials of their students. Put bluntly, 
the students have the potential but are not allowed to express it, at least to the degree they are able to do so when outside of school. The participants of the present study expressed the most creative activity when at home. This may in part reflect their age, culture, or the particular domains measured by the version of the CAAC used here. During the teenage years, youth may still depend on the resources available when at home for certain creative activities. If so, it would be reasonable to determine which resources are relevant and then ensure that they are also available when the youth are at school. Along the same lines, the measures used here were note designed to pinpoint specific processes that might explain the differences among settings, but given the creativity expressed at home it may be that schools should adopt some of the characteristics of home-life, if they want to support the creativity of students. Admittedly some things, such as the small adult:child ratio, is not possible at school.

These findings build on Runco et al. (2017) where creative activity at school was significantly lower than the outside of the school. The present study was more precise than earlier research and distinguished creative activity at home from other outside creative activities. One way to explain the difference between Home and School is that activity at is more structured and planful than Home and accommodating students' individualized interests is much harder in a larger group like in school than at Home. The literature on creative environment (Amabile et al., 1996; Ekvall \& Ryhammar, 1999) emphasizes the importance of freedom, risk-taking, availability of the resources, and idea time, and these are all likely to be more challenging to demonstrate in school than home. Further, the culture of standardized testing and expected evaluation may put much pressure on teachers and students, and the time left for creative activities and accomplishments could easily be limited (Hennessey, 2015; Hondzel, 2014; Niu \& Sternberg, 2003). 
This view of resources could also explain why the mean scores for school were higher than those from outside. When Outside students do have a very large number of choices, and theoretically such a breadth of options could allow creative behavior. Then again, it may be that there is too much latitude Outside. The relatively low scores of the CAAC Outside scale could reflect the fact that the activities on the CAAC were slanted away from the spontaneity and improvisation that probably characterizes Outside, everyday creativity. Research in progress is using a newer CAAC scale, namely one that better assesses everyday creativity. It includes crafts and games and more spontaneous, less format creative activities. The objective of that in progress research is to re-examine how everyday creativity is related to that which is expressed in formal settings (school, work).

Results indicated that both CA\&V and RIBS were positively related to CAAC, but the correlation was higher for RIBS compared to CA\&V. The RIBS measures the frequency of one's ideational behaviors in everyday situations. A person with a higher RIBS score will, for example, have more ideas for a movie title or think of more routes to go home after work. This capacity is not only related to divergent thinking but also creative activities and achievements. Put differently, the correlation between the RIBS and CAAC represent the pathway from the ideation to the implementation of creative skill. CAAC and RIBS represent implementation and ideation aspects of creativity, respectively. The CA\&V was also positively related to CAAC. People with a more positive attitudes and values toward creativity are more likely to engage in more creative activities. It may be that positive values are both an outcome and antecedent of creative activity. Those who hold more positive attitudes and values toward creativity tend to engage in creative activities more because the creative experience has intrinsic value to them. Alternatively, people engaging in creative activity may develop positive attitudes and values toward creativity because 
the activity was rewarding and fulfilling compared to another who apprehend it.

The regression result involving parental education was interesting. It could be that affluent parents overschedule their children so there is little free time for creative and nonacademic activities. There is little research on this topic although findings from Kim and Hua (2019) may be relevant. They reported "Chinese children tend to be more academically successful but less creative than American children." Kim and Hua (2019) attributed such a difference to cultural expectations, beliefs, and values. They concluded "Chinese parenting is shaped by Confucian principles, which emphasize conformity, unquestioned authority, and filial piety through academic success. These tend to impact children's academic achievement positively but creativity development negatively."

This brings us to the limitations of this study and future directions. The most important limitation is probably with the sample. The particulars of this version of the CAAC is also a consideration. Future research should examine relationships of creative activity in different settings with other samples and perhaps with a CAAC that includes additional domains. For now, there is tentative evidence about creative activity varying from one setting to another, but additional research should be conducted before broad generalizations are offered. 


\section{References}

Abdulla Alabbasi, A. M., Tadik, H., Acar, S., \& Runco, M. A. (2021). Birth order and divergent thinking: A meta-analysis. Creativity Research Journal, 1-16. https://doi.org/10.1080/10400419.2021.1913559

Acar, S., \& Runco, M. A. (2014). Assessing associative distance among ideas elicited by tests of divergent thinking. Creativity Research Journal, 26(2), 229-238. https://doi.org/10.1080/10400419.2014.901095

Acar, S., Burnett, C., \& Cabra, J. F. (2017). Ingredients of creativity: Originality and more. Creativity Research Journal, 29(2), 133-144. https://doi.org/10.1080/10400419.2017.1302776

Amabile, T. M., Conti, R., Coon, H., Lazenby, J., \& Herron, M. (1996). Assessing the work environment for creativity. Academy of Management Journal, 39(5), 1154-1184. https://doi.org/10.5465/256995

An, D., \& Runco, M. A. (2016). General and domain-specific contributions to creative ideation and creative performance. Europe's Journal of Psychology, 12(4), 523. https://doi.org/10.5964/ejop.v12i4.1132

Ekvall, G., \& Ryhammar, L. (1999). The creative climate: Its determinants and effects at a Swedish university. Creativity Research Journal, 12(4), 303-310. https://doi.org/10.1207/s15326934crj1204_8

Hair, J. F. Jr., Anderson, R. E., Tatham, R. L. \& Black, W. C. (1995). Multivariate data analysis (3rd ed). Macmillan.

Hennessey, B. A. (2015). If I were Secretary of Education: A focus on intrinsic motivation and creativity in the classroom. Psychology of Aesthetics, Creativity, and the Arts, 9(2), 187- 
192. https://doi.org/10.1037/aca0000012

Hocevar, D. (1981). Measurement of creativity: Review and critique. Journal of Personality Assessment, 45(5), 450-464. https://doi.org/10.1207/s15327752jpa4505_1

Hocevar, D., \& Bachelor, P. (1989). A taxonomy and critique of measurements used in the study of creativity. In J. A. Glover, R. R. Ronning, \& C. R. Reynolds (Eds.), Handbook of creativity (pp. 53-75). Plenum Press. (This chapter is a revised and updated version of an article that appeared in the "Journal of Personality Assessment," 1981, 45, 450-464)

Holland, J. L. (1961). Creative and academic performance among talented adolescents. Journal of Educational Psychology, 52(3), 136-147. https://doi.org/10.1037/h0044058

Holland, J. L., \& Richards, J. M., Jr. (1965). Academic and nonacademic accomplishment: Correlated or uncorrelated? Journal of Educational Psychology, 56(4), 165-174. https://doi.org/10.1037/h0022402

Hondzel, C. D. (2014). Accountable to whom? Teacher reflections on the relationship between creativity and standardized testing in Ontario. Critical Education, 5(3). https://doi.org/10.14288/ce.v5i3.183265

Jauk, E., Benedek, M., \& Neubauer, A. C. (2014). The road to creative achievement: A latent variable model of ability and personality predictors. European Journal of Personality, 28(1), 95-105. https://doi.org/10.1002/per.1941

Kaufman, J. C. (2012). Counting the muses: Development of the Kaufman Domains of Creativity Scale (K-DOCS). Psychology of Aesthetics, Creativity, and the Arts, 6(4), 298-308. https://doi.org/10.1037/a0029751 
Kim, K-H.. \& Hua,Y.(2019). How Cultural Parenting Impacts Children's Academics and Creativity. Creativity. Theories-Research-Applications, 6, 198-222. https://doi.org/10.1515/ctra-2019-0012

Kock, N. (2015). Common method bias in PLS-SEM: A full collinearity assessment approach. International Journal of e-Collaboration, 11(4), 1-10

Ma, H.-H. (2006). A synthetic analysis of the effectiveness of single components and packages in creativity training programs. Creativity Research Journal,18, 435-446. https://doi.org/10.1207/s15326934crj1804_3

Ma, H.-H. (2009). The effect size of variables associated with creativity: A meta-analysis. Creativity Research Journal, 21, 30-42. https://doi.org/10.1080/10400410802633400

Niu, W., \& Sternberg, R. J. (2003). Societal and school influences on student creativity: The case of China. Psychology in the Schools, 40(1), 103-114. https://doi.org/10.1002/pits.10072

Paek, S. H., \& Runco, M. A. (2017). Dealing with the criterion problem by measuring the quality and quantity of creative activity and accomplishment. Creativity Research Journal, 29(2), 167-173. https://doi.org/10.1080/10400419.2017.1304078

Plucker, J. A., Runco, M. A., \& Lim, W. (2006). Predicting ideational behavior from divergent thinking and discretionary time on task. Creativity Research Journal, 18(1), 55-63. https://doi.org/10.1207/s15326934cri1801 7

Rhodes, M. (1961). An analysis of creativity. The Phi Delta Kappan, 42(7), 305-310. https://www.jstor.org/stable/20342603

Rogerson, P. A. (2001). Statistical methods for geography. Sage

Runco, M. A. (1986). Predicting children's creative performance. Psychological Reports, 59(3), 1247-1254. https://doi.org/10.2466/pr0.1986.59.3.1247

Runco, M. A. (2007). A hierarchical framework for the study of creativity. New Horizons in 
Education, 55(3), 1-9.

Runco, M. A. (in press). Assessing creative potential. In M. A. Runco \& S. Acar (Eds.), Handbook of creativity assessment. Elgar.

Runco, M. A., Abdulla, A. M., Peak, S., Aljasim, F. A., \& AlSuwaidi, H. N. (2016). Which test of divergent thinking is best? Creativity. Theories-Research-Applications, 3, 4-18. https://doi.org/10.1515/ctra-2016-0001

Runco, M. A., Acar, S., Campbell, W. K., Jaeger, G., McCain, J., \& Gentile, B. (2016). Comparisons of the creative class and regional creativity with perceptions of community support and community barriers. Business Creativity and the Creative Economy, 2(1), 83-92. https://doi.org/10.18536/bcce.2016.10.2.1.08

Runco, M. A., Acar, S., \& Cayirdag, N. (2017). A closer look at the creativity gap and why students are less creative at school than outside of school. Thinking Skills and Creativity, 24, 242-249. https://doi.org/10.1016/j.tsc.2017.04.003

Runco, M. A., Noble, E. P., \& Luptak, Y. (1990). Agreement between mothers and sons on ratings of creative activity. Educational and Psychological Measurement, 50, 673-680. https://doi.org/10.1177/0013164490503025

Runco, M. A., Plucker, J. A., \& Lim, W. (2001). Development and psychometric integrity of a measure of ideational behavior. Creativity Research Journal, 13(3-4), 393-400. https://doi.org/10.1207/S15326934CRJ1334_16

Simonton, D. K. (1990). History, chemistry, psychology, and genius: An intellectual autobiography of historiometry. In M. A. Runco \& R. S. Albert (Eds.), Theories of creativity (pp. 92-115). Sage.

Simonton, D. K. (2012). Taking the US Patent Office criteria seriously: A quantitative three- 
criterion creativity definition and its implications. Creativity Research Journal, 24(2-3), 97-106. https://doi.org/10.1080/10400419.2012.676974

Weisberg, R. W. (2015). On the usefulness of "value" in the definition of creativity. Creativity Research Journal, 27(2), 111-124. https://doi.org/10.1080/10400419.2015.1030320

Weisberg, R., Pichot, N., Bonetto, E., Pavani, J. B., Arciszewski, T., \& Bonnardel, N. (2021). From explicit to implicit theories of creativity and back: The relevance of naive criteria in defining creativity. The Journal of Creative Behavior. https://doi.org/10.1002/jocb.492 
Table 1

Descriptive Statistics and Bivariate Correlations of the Study Variables

\begin{tabular}{|c|c|c|c|c|c|c|c|c|c|}
\hline & $M$ & $S D$ & 1 & 2 & 3 & 4 & 5 & 6 & 7 \\
\hline 1. CAAC Home & 28.49 & 12.64 & & & & & & & \\
\hline 2. CAAC School & 24.00 & 13.08 & $.721 * *$ & & & & & & \\
\hline 3. CAAC Outside & 17.43 & 13.78 & $.635 * *$ & $.700 * *$ & & & & & \\
\hline 4. WAYMC Home & 4.52 & 3.73 & $-.160 * *$ & $-.237 * *$ & $-.111 * *$ & & & & \\
\hline 5. WAYMC School & 2.36 & 2.33 & -.059 & .059 & $-.082 *$ & $-.234 * *$ & & & \\
\hline 6. WAYMC Other & 1.78 & 2.19 & $.084 *$ & .052 & $.141^{* *}$ & $-.250 * *$ & $-.155 * *$ & & \\
\hline 7. A \& V & 85.03 & 11.66 & $.159 * *$ & $.201 * *$ & $.171^{* *}$ & .009 & .007 & .022 & \\
\hline 8. RIBS & 84.29 & 18.35 & $.419 * *$ & $.411 * *$ & $.351 * *$ & $-.089 *$ & -.015 & $.094 *$ & $.525 * *$ \\
\hline
\end{tabular}

